\title{
Commissioning of CALLISTO spectrometers in Peru and observations of type III Solar Radio Bursts
}

\author{
J.A. Rengifo, ${ }^{a, b}$ V. Loaiza-Tacuri, ${ }^{c, b}$ J. Bazo ${ }^{a, *}$ and W.R. Guevara Day ${ }^{d, b}$ \\ ${ }^{a}$ Sección Física, Departamento de Ciencias, Pontificia Universidad Católica del Perú \\ Av. Universitaria 1801, Lima 32, Peru \\ ${ }^{b}$ Dirección de Astrofísica, Comisión Nacional de Investigación y Desarrollo Aeroespacial, CONIDA \\ Luis Felipe Villarán 1069, Lima, Peru \\ ${ }^{c}$ Observatório Nacional \\ Rua General José Cristino, 77, 20921-400 São Cristóvão, Rio de Janeiro, RJ, Brazil \\ ${ }^{d}$ Facultad de Ciencias Físicas, Universidad Nacional Mayor de San Marcos \\ P.O. Box 14-149, Lima 14, Peru \\ E-mail: jbazo@pucp.edu.pe
}

Two radio spectrometer stations belonging to the e-CALLISTO network were installed in Peru by the Astrophysics Directorate of CONIDA. Given their strategic location near the Equator, it was possible to observe the Sun evenly throughout the whole year and the detector was unique in its time-zone coverage. The receiver located nearby the capital city of Lima took data in the metric and decimetric bands looking for radio bursts. To assess the suitability of the sites and the performance of the antennas, we analysed the radio ambient background and measured their radiation pattern and beam-width. To show the capabilities of the facilities to study solar dynamics in these radio frequencies we have selected and analysed type III Solar Radio Bursts. We have characterised the most common radio bursts with the following mean values: a negative drift rate of $-25.8 \pm 3.7 \mathrm{MHz} / \mathrm{s}$, a duration of $2.6 \pm 0.3 \mathrm{~s}$ and $35 \mathrm{MHz}$ bandwidth in the frequency range of 114 to $174 \mathrm{MHz}$. In addition, for some events, it was possible to calculate a global frequency drift, which on average was $0.4 \pm 0.1 \mathrm{MHz} / \mathrm{s}$.

$37^{\text {th }}$ International Cosmic Ray Conference (ICRC 2021)

July 12 th - 23rd, 2021

Online - Berlin, Germany

\footnotetext{
${ }^{*}$ Presenter
} 


\section{Introduction}

The e-CALLISTO network (Compound Astronomical Low frequency Low cost Instrument for Spectroscopy and Transportable Observatory) $([1,2])$ has more than 170 radio spectrometer stations around the world. Its main scientific goal is to monitor all types of solar radio activities at a wide range of frequencies (i.e. from 45 to $870 \mathrm{MHz}$ ).

Two CALLISTO spectrometers with an LPDA (Logarithmic Periodic Dipole Array) antenna were installed and commissioned [3], between 2012 and 2014, in two sites in Lima, Peru, by the Astrophysics Directorate of CONIDA (Comisión Nacional de Investigación y Desarrollo Aeroespacial). During the operation period, these e-CALLISTO stations were unique in their time-zone (i.e. GMT-5) coverage. Currently, there are more instruments in this time zone and similar stations have been deployed elsewhere (e.g. [4, 5])

We have used the recorded radio data to look for type III Solar Radio Bursts (SRBs), which are common transient bursts. We have found burst candidates from the data taking period and analyzed their spectra, showing the observational capabilities of the facilities. Here we show the results of these observations and their calculated burst parameters. Other type III SRBs have also been characterized using similar e-CALLISTO stations ([6-10]).

\section{Commissioning of e-CALLISTO Stations in Peru}

Two e-CALLISTO stations were installed in Lima, Peru, by the Astrophysics Directorate of CONIDA. CALLISTO NA-06 was commissioned early on in 2012 in San Isidro district at the main offices of CONIDA $\left(-12^{\circ} 06^{\prime} 51^{\prime \prime} \mathrm{S},-77^{\circ} 03^{\prime} 27^{\prime \prime} \mathrm{W}\right)$. This receiver was only a test for the facilities given its intense background noise, since it was located inside the city center. On the other hand, CALLISTO NA-18 in Pucusana district (Punta Lobos) at the CONIDA scientific site (-12'30'18" $\left.\mathrm{S},-77^{\circ} 47^{\prime} 56^{\prime \prime} \mathrm{W}\right)$, was installed later in 2014. This receiver operated between October 2014 and August 2016 providing data to the e-CALLISTO network. However, after 2016 it was no longer maintained. There are plans to get the instruments back into operation.

Each station was implemented with an LPDA antenna with 23 elements covering the 70-1000 $\mathrm{MHz}$ range. The front-end electronics comprised a low noise amplifier ZX60-33LN-S+ from Mini-Circuits, connected through a coaxial wire to the e-CALLISTO spectrometer ([1]). Data were transferred via a RS-232 cable to a computer. The spectrometer operates in the $45-870 \mathrm{MHz}$ bandwidth. For 200 channels, the time resolution is $0.25 \mathrm{~s}$ and the integration time $1 \mathrm{~ms}$.

To assess the suitability of the sites the Radio Frequency Interference (RFI) was analyzed. We seek to identify radio-quite zones for radio-astronomy. The interference measurements were done with a simple dipole. We used a spectral analyzer INSTEK GSP-GW-830 for frequencies from 9 $\mathrm{kHz}$ to $3 \mathrm{GHz}$, with more power from $45 \mathrm{MHz}$ to $870 \mathrm{MHz}$.

In Fig. 1 we show the RFI intensity taken in 2014 as a function of frequency for both sites. The interference in San Isidro is very intense, because it is located inside the city, where there are several telecommunication activities and transmitters. Thus, this receiver could not monitor solar radio phenomena due to its large background during the whole data taking period. Instead, the Pucusana site has lower background noise, since it is located in the outskirts of the city and has a 


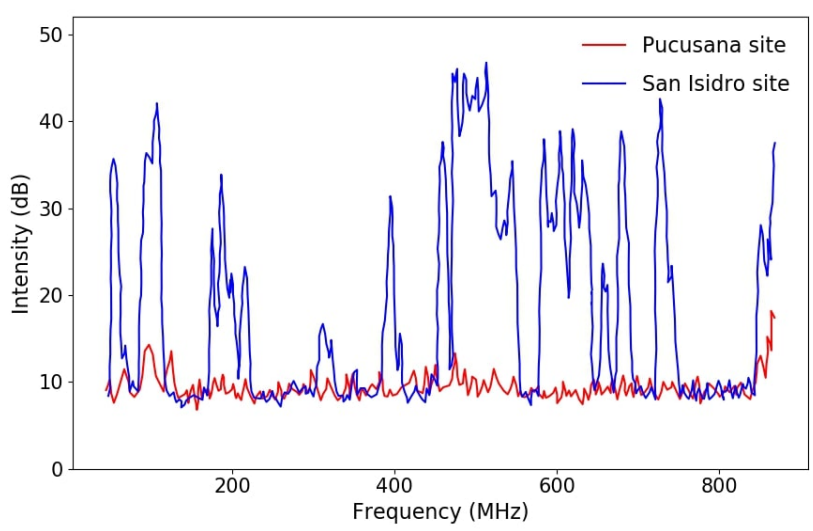

Figure 1: Radio Frequency Interference intensity as a function of the frequency, measured with a dipole at San Isidro (blue) and Pucusana (red) sites.

natural terrain shielding given by the surrounding hills. Therefore, we consider the Pucusana site to be a good option for SRBs observations.

The sensitivity of the LPDA antennas were characterized by determining their radiation pattern and beamwidth. Each antenna was connected to a spectrum analyzer to measure the reception maximum power. The reference signal, centered at $481.76 \mathrm{MHz}$, was emitted by the repeater antenna of the National Institute of Radio and Television of Peru. Once the signal was found, we oriented the antenna such that it provided the maximum power and from that point, we rotated the antenna a full $360^{\circ}$ sweep, in $10^{\circ}$ steps.

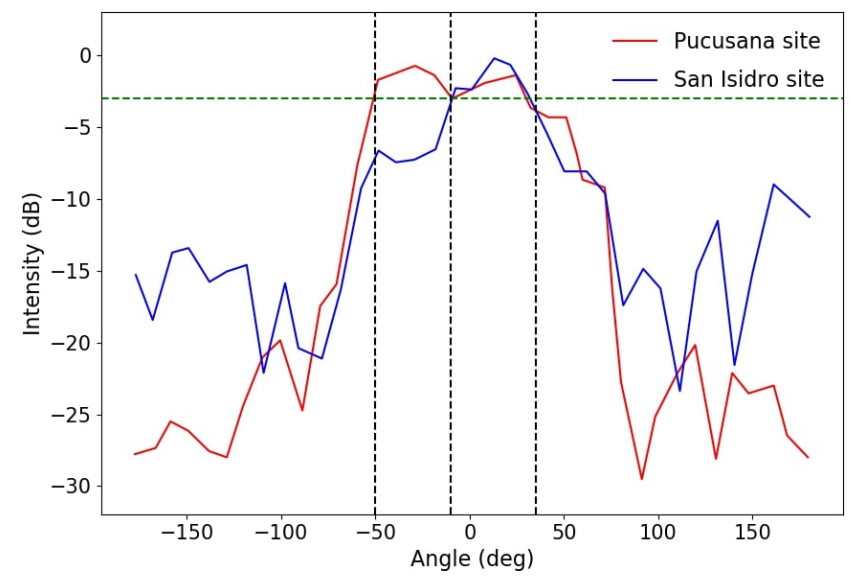

Figure 2: LPDA antennas radiation patterns measured with the spectral analyzer in San Isidro site (blue) and Pucusana site (red). The dashed black lines mark the beam-width at $-3 \mathrm{~dB}$ (green dashed line).

In Fig. 2 we show the measurements of the radiation patterns at both sites. The beam-width, the angle between the points where the signal strength is half of its maximum radiated power $(-3$ $\mathrm{dB}$ ), was $41^{\circ}$ for the San Isidro site and $43^{\circ}$ for the Pucusana site. 


\section{Radio Observations with the Peruvian e-CALLISTO station at Pucusana}

The e-CALLISTO station at the Pucusana site (i.e. Punta Lobos) took data from October 10th, 2014 until August 3th, 2016, with few missing data. These data are stored in the e-CALLISTO network server [11], which has the spectra of all instruments of the network. For this study, we searched for radio bursts from the data taken by this station (i.e. CONIDA_PL in the server).

After searching the whole dataset for radio bursts, we selected the most significant signals for this analysis, comprising a set of twelve events. These took place on different days between December 2014 and June 2015. In Fig. 3 we show the dynamical spectra of these recorded radio signals, which we consider are type III SRBs with their characteristic shapes, as single or group bursts. The events are ordered using Roman numbers. The first two events are found at a higher frequency range, from 178 to $433 \mathrm{MHz}$ and the remaining events are in the range from 114 to 174 MHz. Details of the processing and cleaning can be found in [3].

For each dynamical spectra the most intense or isolated radio burst is chosen for a more detailed analysis. The observation date and time, significance cut, frequency range, drift rate and duration are listed in Table 1. In addition, for the cases where it was possible, we have calculated a global frequency drift for group bursts.

\begin{tabular}{||ccccccc||}
\hline SRB & $\begin{array}{c}\text { Date } \\
(\mathrm{UT})\end{array}$ & $\begin{array}{c}\text { cut } \\
(\sigma)\end{array}$ & $\begin{array}{c}\text { Frequency Range } \\
(\mathrm{MHz})\end{array}$ & $\begin{array}{c}\text { Drift } \\
(\mathrm{MHz} / \mathrm{s})\end{array}$ & $\begin{array}{c}\text { Duration } \\
(\mathrm{s})\end{array}$ & $\begin{array}{c}\text { Global Drift } \\
(\mathrm{MHz} / \mathrm{s})\end{array}$ \\
\hline I & $2014 / 12 / 21-12: 44: 39$ & 2.5 & $411-433$ & $-84.0 \pm 28.2$ & $1.39 \pm 0.02$ & $\mathrm{~s}$ \\
II & $2014 / 12 / 21-13: 05: 08$ & 1 & $178-196$ & $-41.4 \pm 5.4$ & $1.32 \pm 0.03$ & $\mathrm{~s}$ \\
III & $2015 / 01 / 14-13: 28: 47$ & 3.5 & $122-140$ & $-15.5 \pm 0.5$ & $3.12 \pm 0.16$ & $0.55 \pm 0.35$ \\
IV & $2015 / 01 / 14-13: 32: 10$ & 3 & $118-140$ & $-14.0 \pm 3.3$ & $3.77 \pm 0.27$ & $*$ \\
V & $2015 / 01 / 14-14: 05: 02$ & 2.5 & $131-157$ & $-35.2 \pm 1.8$ & $2.11 \pm 0.08$ & $0.20 \pm 0.14$ \\
VI & $2015 / 01 / 25-13: 18: 54$ & 2 & $122-174$ & $-39.2 \pm 2.4$ & $1.50 \pm 0.02$ & $\mathrm{~s}$ \\
VII & $2015 / 01 / 26-11: 44: 01$ & 4 & $127-170$ & $-23.8 \pm 4.6$ & $4.48 \pm 1.66$ & $0.51 \pm 0.17$ \\
VIII & $2015 / 01 / 26-11: 50: 37$ & 3 & $127-170$ & $-30.2 \pm 2.8$ & $2.91 \pm 0.03$ & $*$ \\
IX & $2015 / 01 / 26-15: 37: 27$ & 1.5 & $123-140$ & $-18.8 \pm 3.9$ & $2.05 \pm 0.03$ & $\mathrm{~s}$ \\
X & $2015 / 02 / 01-12: 59: 26$ & 2 & $127-149$ & $-18.5 \pm 1.4$ & $1.88 \pm 0.03$ & $0.14 \pm 0.02$ \\
XI & $2015 / 02 / 01-14: 36: 38$ & 2.5 & $127-170$ & $-25.3 \pm 5.8$ & $2.99 \pm 0.18$ & $\dagger$ \\
XII & $2015 / 06 / 30-17: 17: 26$ & 1 & $114-170$ & $-23.1 \pm 1.5$ & $1.49 \pm 0.02$ & $\mathrm{~s}$ \\
\hline
\end{tabular}

Table 1: Radio Bursts characteristics corresponding to the numbering shown in Fig. 3. Note: for the global drift s stands for single (isolated) bursts (i.e. no global drift), * represents group bursts with a weak second signal where no global drift could be calculated and $\dagger$ corresponds to group bursts with constant frequency.

The burst frequency range is defined where the signal is above the background given a significance cut. We compare the measured spectra with the mean intensity in a 15-minute interval from one day before without signal (i.e. background $(b)$ ). We calculate for each frequency the average intensity $(n)$ over the time interval of the burst (i.e. $\approx 2 \mathrm{~s}$ ). Using the significance $\left(S=\frac{n-b}{\sqrt{b}}\right)$, we apply a cut at different $\sigma$ s to select the frequency signal range to calculate the drift rate. The significance cut depends on the strength of the signal and has a lower limit of $1 \sigma$ and mean of $2.4 \sigma$. 


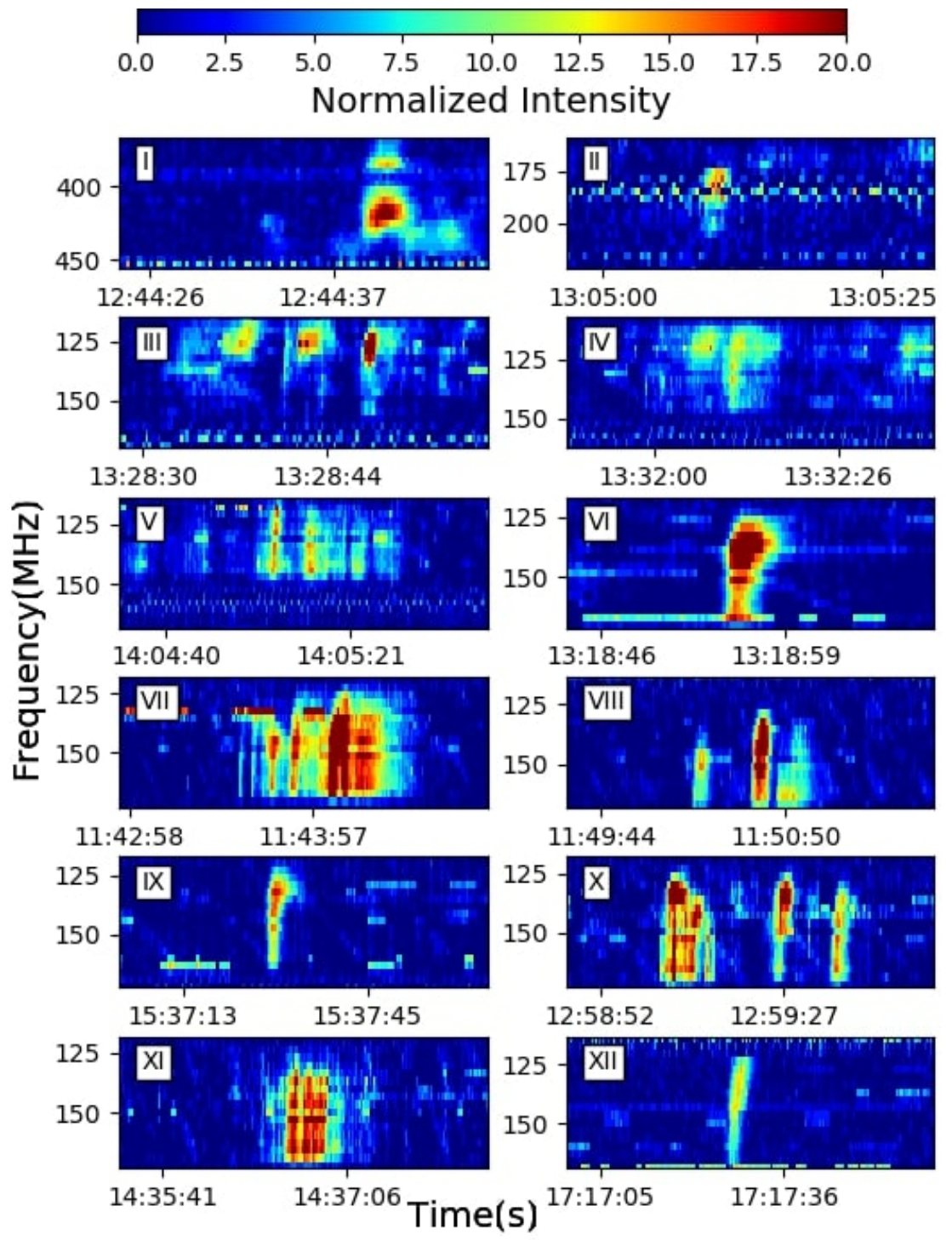

Figure 3: Dynamical spectra of type III SRB events taken with CALLISTO NA-18 station at the Pucusana site. Data are centered in the frequency and time ranges of the signal. Further details are given in Table 1.

\subsection{Type III Solar Radio Bursts Parameters}

The recorded radio spectrum is used to study the characteristics of type III SRBs. These common radio transient emissions are characterized by a fast drift from higher to lower frequencies over time ([12],[13]). They can appear isolated, in groups or in storms and can be accompanied by a second harmonic. The duration for single bursts is $1-3 \mathrm{~s}$, for bursts in groups it ranges from 1 to 5 minutes and in storms from minutes to hours ([4]). These radio emissions are produced by non-thermal electrons accelerated in the solar corona. They are considered to originate near to the particle acceleration regions during solar flares ([14]).

A feature of SRBs is their frequency drift rate $D=d f / d t$, which is an indication of fast electron 
beams moving in the solar corona. The drift rate is the positive or negative displacement of the frequency representing the burst peak flux in a time interval ([12]).

To calculate $D$ we first create the light curves of each measured channel (frequency) of the spectrometer, excluding the frequencies with an intensity below a given significance depending on the strength of the signal, as discussed before. For each light curve, we fit a Gaussian profile around the peak flux. From the fit we take the time corresponding to the center of the Gaussian, which together with the specific frequency forms an ordered pair. This is drawn in a scattered plot as seen in Fig. 4 for SRB VIII, as an example. We observe a linear dependency representing the frequency drift rate, which is estimated from a linear regression of these points.

To obtain the observed duration of the bursts, we get the full width at half maximum (FWHM) of each light curve peak Gaussian fit. Then, the duration is taken as the average of these FWHMs ([15]) from all frequencies profiles for each SRB.

We calculated the global frequency drift for solar events with a group of consecutive bursts, defined in [16], as the variation of the central burst frequency during the time of the event, called drifting pulsating structure [17]. In order to choose the central frequency of each burst, we follow the same procedure described before, applying a different significance cut to each burst in the group, depending on the signal intensity. Some studies [17] suggest that these global drifts occur because the plasmoids, semi-closed magnetic field structures, move in the stratified solar atmosphere.

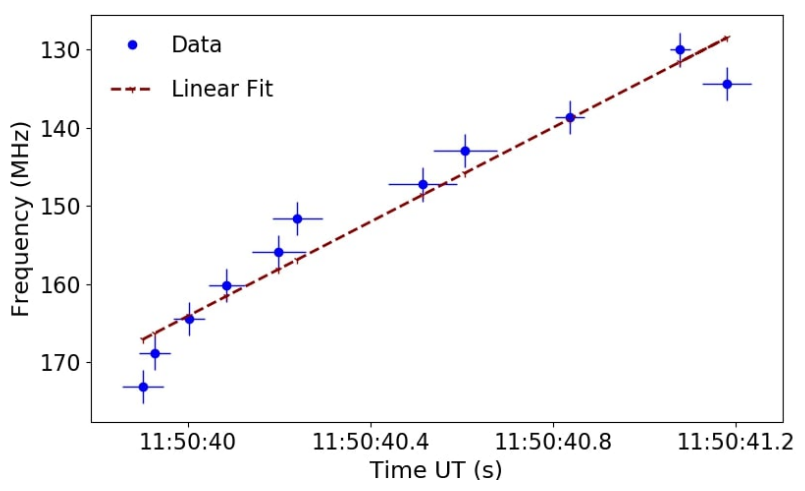

Figure 4: Frequencies of the peak flux for each LC and associated times for SBR VIII. The red dashed line represents the linear regression $\left(\chi_{\text {red }}^{2}=13.8\right)$, which in this case shows a negative drift rate.

All observed SRBs have negative drift rates. The first SRB shown in Fig. 3 appears in a higher frequency range from 411 to $433 \mathrm{MHz}$ with drift rate $\mathrm{D}=-84.0 \pm 28.2 \mathrm{MHz} / \mathrm{s}$. The second SRB is between $178-196 \mathrm{MHz}$ and has a drift of $-41.4 \pm 5.4 \mathrm{MHz} / \mathrm{s}$. All other observed SRBs are between 114 to $174 \mathrm{MHz}$ and their calculated drifts have a mean of $-25.8 \pm 3.72 \mathrm{MHz} / \mathrm{s}$. Negative drift rates are standard for type III SRBs. This can be attributed to electron beams moving away from the solar surface up to the high corona along open field lines, from regions of high to low densities ([18]). The duration of the higher frequency range burst was $1.39 \pm 0.02 \mathrm{~s}$, for the middle frequency range it was $1.32 \pm 0.03 \mathrm{~s}$, while for the lower frequencies it was $2.6 \pm 0.25 \mathrm{~s}$. The mean bandwidth was $28 \mathrm{MHz}$ for the first range, $18 \mathrm{MHz}$ for the second range and $35 \mathrm{MHz}$ for the third range.

In addition, we calculated a characteristic property of plasmoids, the global frequency drift, for group bursts, excluding isolated events and weak signals, listed in Table 1. We found four group 
bursts, out of the twelve SRB events, with a positive drift with mean $0.4 \pm 0.1 \mathrm{MHz} / \mathrm{s}$. These low values indicate that the drift varies slowly.

\section{Conclusions}

Two e-CALLISTO stations have been installed and commissioned in Lima, Peru. During the data taking period of the analyzed receiver, the Peruvian e-CALLISTO detector was unique in its time-zone coverage. We have calibrated the antennas measuring their radiation pattern and beamwidth. We have also analyzed the ambient background noise, showing that the San Isidro station, installed earlier in 2012 for tests purposes, had an intense RFI, not suitable for radio observations. However, the Pucusana station, installed in 2014, had a lower background due to the natural terrain shielding and distance to the city.

We took data looking for SRBs candidates between October 10th, 2014 and August 3th, 2016 at the Pucusana site. We have shown that this station was able to observe type III SRB events in the metric and decimetric bands. Nevertheless, only twelve radio bursts have been identified with a significance larger than $1 \sigma$ in the whole dataset. The most common type III SRB had been characterized with the following values: they are located in the frequency range between 114 to 174 $\mathrm{MHz}$, they have a negative drift rate of $-25.8 \pm 3.7 \mathrm{MHz} / \mathrm{s}$, a duration of $2.6 \pm 0.3 \mathrm{~s}$ and $35 \mathrm{MHz}$ bandwidth. For some events showing group bursts, it was possible to calculate a global frequency drift with mean $0.4 \pm 0.1 \mathrm{MHz} / \mathrm{s}$.

\section{Acknowledgements}

J. R. appreciates the support from the Peruvian National Council for Science, Technology and Technological Innovation scholarship under Grant 23-2015-FONDECyT. J. B. thanks the Dirección de Gestión de la Investigación (DGI - PUCP) for funding under Grant No. DGI-2019-3-0044.

We thank the Institute for Data Science FHNW Brugg/Windisch, Switzerland for providing data of the e-CALLISTO network. We specially thank C. Monstein for his help and suggestions in the analysis of the e-CALLISTO data.

\section{References}

[1] A.O. Benz, C. Monstein, and H. Meyer. CALLISTO - a new concept for solar radio spectrometers. Solar Physics, 226(1):143-151, 2005.

[2] A.O. Benz, C. Monstein, H. Meyer, P.K. Manoharan, R. Ramesh, A. Altyntsev, A. Lara, J. Paez, and K.-S. Cho. A world-wide net of solar radio spectrometers: e-CALLISTO. Earth, Moon and Planets, 104(1-4):277-285, 2009.

[3] J. Rengifo, V. Loaiza-Tacuri, J. Bazo, and W. R. Guevara-Day. Research in Astronomy and Astrophysics, 21(6), June 2021.

[4] N. Prasert, A. Phakam, K. Asanok, P. Jaroenjittichai, A. Chumthong, and T. Thongmeearkom. CALLISTO spectrometer for solar radio bursts monitoring in Chiangmai. Journal of Physics: Conference Series, 1380(1), 2019. 
[5] A. Zavvari, M.T. Islam, R. Anwar, Z.Z. Abidin, M.F. Asillam, and C. Monstein. Analysis of radio astronomy bands using CALLISTO spectrometer at Malaysia-UKM station. Experimental Astronomy, 41(1-2):185-195, 2016.

[6] N.M. Ansor, Z.S. Hamidi, and N.N.M. Shariff. Characterization of type III and IV solar radio bursts from e-CALLISTO. Journal of Physics: Conference Series, 1349(1), 2019.

[7] Z.S. Hamidi, N. Ramli, and N.N.M. Shariff. Statistical analysis of solar radio burst type III and type IV with relation to solar activities. Journal of Physics: Conference Series, 1152(1), 2019.

[8] Z.S. Hamidi, N.H. Zainol, M.O. Ali, S.N.U. Sabri, N. Husien, N.N.M. Shariff, M.S. Faid, and C. Monstein. Signal detection of the solar radio burst type III based on the CALLISTO system project management. ICIMSA 2016, 2016.

[9] M.O. Ali, S.N.U. Sabri, Z.S. Hamidi, N. Husien, N.N.M. Shariff, N.H. Zainol, M.S. Faid, and C. Monstein. E-CALLISTO network system and the observation of structure of solar radio burst type III. ICIMSA 2016, 2016.

[10] N. Ramli, Z.S. Hamidi, Z.Z. Abidin, and S.N. Shahar. The relation between solar radio burst types II, III and IV due to solar activities. International Conference on Space Science and Communication, IconSpace, pages 123-127, 2015.

[11] FHNW Windisch. Observations of generation aos, argos, phoenix-3, phoenix-4 and e-callisto, 2020. Accessed: 2020-11-13.

[12] H. Reid, A. Sinclair, and H. Ratcliffe. A review of solar type III radio bursts. Research in Astronomy and Astrophysics, 14(7):773-804, Jul 2014.

[13] S. Suzuki and G. A. Dulk. Bursts of type III and type V. Solar Radiophysics, pages 289-332, 1985.

[14] H. Mészárosová, M. Karlický, H. S. Sawant, F. C. R. Fernandes, J. R. Cecatto, , and M. C. de Andrade. Solar decimetric type III bursts in semi-closed magnetic field structures. Astronomy and Astrophysics, 484(2):529 - 536, 2008.

[15] P. Zhang, S. Yu, E.P. Kontar, and C. Wang. On the source position and duration of a solar type III radio burst observed by LOFAR. Astrophysical Journal, 885(2), 2019.

[16] B. L Tan. Observable parameters of solar microwave pulsating structure and their implications for solar flare. Solar Physics, 253:117-131, 2008.

[17] M Bárta, M. Karlický, and R. Žemlicka. Plasmoid dynamics in flare reconnection and the frequency drift of the drifting pulsating structure. Solar Physics, 253:173-189, 2008.

[18] P.A. Robinson and A.O. Benz. Bidirectional type III solar radio bursts. Solar Physics, 194(2):345-369, 2000. 